\title{
Study on the economic dispatch of regional integrated energy system based on master-slave game
}

\author{
Yongli Wang ${ }^{1}$, Yang $\mathrm{Ma}^{1}$, Yuze $\mathrm{Ma}^{1}$, Suhang Yao ${ }^{1, *}$, Siyi Tao ${ }^{1}$, Hekung Shen ${ }^{1}$, Xin Chen ${ }^{1}$ \\ ${ }^{1}$ North China Electric Power University, Beijing, 102206, China
}

\begin{abstract}
Traditional demand response technologies optimize the load profile by cutting or shifting the electrical load, but they affect the customer's energy experience, and thus the customer's motivation to participate in demand response and load controllability are limited. For community integrated energy operators, the study of integrated customer demand response can explore their dispatch potential. Based on consumer psychology, we study the alternative energy use behavior of residential customers, establish a customer optimization model and a community integrated energy operator optimization model, and set up a Stackelberg master-slave game model with the operator as the leader and the customer as the follower. The simulation case verifies that the utilization of load-side multi-energy complementary characteristics and integrated demand response behavior can effectively improve the utilization of renewable energy and the efficiency of integrated energy operators.
\end{abstract}

\section{Introduction}

The increasingly serious energy and environmental problems of the earth have attracted widespread attention from all walks of life, and the only solution nowadays is to vigorously develop renewable energy and integrated energy internet, improve energy utilization efficiency, follow the path of sustainable development, and reduce non-renewable energy consumption. As a result, the integrated energy system [1], which has excellent attributes of energy gradient utilization, multi-energy complementation and high energy utilization rate, and the demand-side response [2], which has positive significance for renewable energy consumption, have become hot spots for many scholars' research in recent years.

Adjusting load distribution through demand-side management is an important means to effectively increase the system wind power consumption rate. A lot of research has been conducted on the role of demand-side management in wind power consumption, the uncertainty of demand response DR (Demand Response) and the application of master-slave game in grid scheduling. The paper [3] proposes a two-stage scheduling method for wind power consumption in the day-ahead and intra-day phases by constructing a comprehensive electric and thermal system scheduling model that includes thermal storage, CHP and DR resources: in the day-ahead scheduling phase, units, thermal storage devices and tariffbased DR cooperate to consume the short-term forecasted wind power output; in the intra-day scheduling phase, units and incentive-based DR cooperate to consume the ultra-short-term forecasted wind power output. In the literature [4], considering the prediction error of new energy output and the characteristics of load DR, a multitimescale scheduling model of power system with wind power containing three stages: day-ahead, intra-day and real-time is constructed to improve the system wind power consumption capacity and reduce the system wind abandonment. In the paper [5], with the objectives of smoothing load fluctuations and reducing vehicle owners' electricity bills, a multi-objective optimization model of wind-electric vehicle cooperative scheduling with DR is established by guiding electric vehicles to the grid through price mechanisms and coordinating and optimizing generation-side resources to consume wind power. The literature [6] applies Stackelberg's dynamic game theory to establish a dynamic game model for the virtual power plant bidding problem based on the independence of property rights of the internal subjects of the virtual power plant and the existence of sequential action order between electricity price bidding and power bidding. The above literature provides references for studying the role of demand-side management in improving the level of wind power consumption and the application of master-slave game model in power systems, but none of them considers the influence of the master-slave game role between the grid as the main demand-side management tariff-setting party and the customer side as the tariff recipient on reaching each other's optimization goals, nor does it consider the influence of DR uncertainty on the load side on the wind power consumption rate.

In this paper, the customer load is divided into purchased energy load and direct energy use load, and the residential customers' alternative energy use behavior is studied based on consumer psychology. The community integrated energy operator and residential consumers are taken as the research objects, and the user-optimized

\footnotetext{
* Corresponding author: 120202206303@ncepu.edu.cn
} 
energy use model is established with the objective of consumer surplus, and the regional integrated energy system optimization model is established with the objective of profit, and the Stackelberg master-slave game model is established with the regional integrated energy system as the leader and the consumers as the followers, and its equilibrium solution is proved to exist and unique. The user optimization model is replaced by its KKT condition, and the game model is transformed into a single-layer optimization model to solve. The simulations show that it is meaningful to fully exploit the multi-energy complementary characteristics of users and to consider the influence of energy price on the alternative energy use of users for the operational efficiency of regional integrated energy systems, the optimal operation of community integrated energy systems and the improvement of renewable energy utilization.

\section{Integrated regional energy and customer-side energy systems}

(1) Based on this modeling of the residential user groups in the community, the power balance is shown below.

$$
\left\{\begin{array}{l}
P_{t}^{b u y}=P_{t}+P_{t}^{a c}+P_{t}^{w h} \\
H_{t}^{b u y}=H_{t}-P_{t}^{a c} \cdot \ell_{a c}-P_{t}^{w h} \cdot \ell_{w h}
\end{array}\right.
$$

where: $P_{t}^{b u y}$ is the electricity purchased by the customer from the regional integrated energy operator; $H_{t}^{b u y}$ denotes heat purchase; ${ }^{\ell}{ }_{a c}, \ell_{w h}$ denotes the heat production coefficient of air conditioners and the heat production coefficient of electric water heaters,,$P_{t}$ 、

$H_{t}$ is the direct electricity and heat load.

Considering the participation of load aggregators [6] in the electricity DR, this paper divides the residential direct electricity load into baseline load, levelizable load and curtailable load. The models corresponding to the three types of loads are as follows.

$$
P_{b l, t}=L_{b l, t}, \int_{0}^{T} P_{t l, t} d t=\int_{0}^{T} L_{t l, t} d t, P_{r l, t}^{\min } \leq P_{r l, t} \leq P_{r l, t}^{\max }
$$

where: $P_{b l, t} 、 P_{t l, t}$ and $P_{r l, t}$ are the baseline load, levelable load and curtailable load power, respectively; $L_{t l, t}$ is the total power consumption of levelable electric load before demand response.

(2) Integrated demand response model based on consumer psychology

According to the different response mechanisms can be divided into price-based DR and incentive-based DR, and most studies describe price-based DR as the priceelastic demand curve shown in Figure 1. As seen in Figure 1, when the uncertainty of price-based DR is not accounted for, the amount of change in DR is

$$
\Delta p_{t}^{\mathfrak{I}}=\eta_{t}^{\mathfrak{J}} \alpha_{t}^{\Delta c} P_{b l, t}
$$

where $\Delta p_{t}^{\mathfrak{I}}$ is the predicted value of the change in price-based DR at moment t; $\alpha_{t}^{\Delta c}$ is the rate of change of electricity price at moment $\mathrm{t} ; \eta_{t}^{\mathfrak{\Im}}$ and is the load elasticity coefficient at moment $t$.

In the actual response, price-based DR follows the principle of voluntary participation of customers, so the load response is affected by the tariff, but also by various non-economic factors such as communication delays, component failures, unexpected events, and customer response reputation [7]; at the same time, due to the influence of the next day's weather conditions, customer production arrangements, and uncertainty of real-time load data, the baseline load forecasting for customers also There is a certain degree of uncertainty in the baseline load forecasting. The uncertainty of price-based DR is mainly the uncertainty of baseline load and price elasticity of demand curve.

\section{Mathematical model of master-slave game}

In this paper, from the perspective of a regional integrated energy operator, the price curve of energy sales is optimized with the goal of maximizing profits by considering the alternative energy use and demand response of customers. The DNO needs to consider the response and decision of the customers after receiving the price information, and then update its own optimization decision according to the customers' decision in order to achieve the ultimate goal of profit maximization. This optimization problem can be quantified and characterized by building a Stackelberg master-slave game model, and then the optimal strategy of the RIO can be obtained by solving the Nash equilibrium solution of the game model.

\subsection{Upper-level optimization problem}

The regional integrated energy operator is the leader of the game, the set of strategies is the price of energy sold at each moment, and the optimization objective of the regional integrated energy operator is to maximize profit.

$$
\begin{aligned}
\operatorname{maxR}= & \int_{1}^{2} \int_{1}^{T}\left(\mathrm{c}_{j, t} \cdot P_{t}^{b u y}\right) d t d j-\sum_{i=1}^{z} C_{i, t} \\
& \mathrm{c}_{j, t}^{\min } \leq \mathrm{c}_{j, t} \leq \mathrm{c}_{j, t}^{\max }
\end{aligned}
$$

where ${ }^{\mathrm{c}_{j, t}}$ denotes the price strategy for energy sales at each moment; to ensure that the operator is profitable and attractive to customers, $\mathrm{c}_{j, t}^{\min }$ it is not lower than the operator's marginal cost and $\mathrm{c}_{j, t}^{\max }$ not higher than the external energy grid price.

\subsection{Lower level optimization model}

At a given energy price, the user-optimized energy use objective function is the maximum consumer surplus, i.e., the user's energy use utility minus the energy purchase cost.

$$
\max U=\int_{1}^{T}\left(\int_{1}^{5} f\left(P_{b l, x}, P_{t l, x}, P_{r l, x}\right) d x-\int_{1}^{2}\left(\mathrm{c}_{j, t} \cdot P_{t}^{b u y}\right) d j\right) d t
$$


where: $j$ is the purchased energy type; $\mathbf{c}$ is the energy price at time $\mathrm{t}$; $P_{t}^{b u y}$ is the energy power purchased by the user.

The user as a follower of the game, its own energy use behavior after accepting the energy price signal, the user alternative energy use behavior through the consumer psychology principle expression, the baseline electric load and direct heat load utility is constant, at this time the user's strategy set for the levelable electric load and can cut the electric load of energy use $\left(P_{b l, x}, P_{t l, x}, P_{r l, x}\right)$.

\subsection{Model solving}

A sufficient condition for the existence of a unique equilibrium solution to the stackelberg game is that the leader's objective function is a nonempty, continuous function of its set of strategies and the follower's objective function is a continuous convex concave function of its set of strategies [9]. The unique equilibrium solution of the above game model is proved as follows: it is shown that the community operator as a leader has a set of strategies with energy valence $\left\{{ }^{\mathrm{c}_{j, t}}\right\}$ at each moment, and the first term of its objective function is a continuous linear function of energy valence, and the second term $\mathrm{C}$ varies only with the load value and has a unique optimal solution with respect to the load value, but is a constant for the set of strategies $\left\{\mathrm{c}_{j, t}\right\}$, thus proving that the operator's objective function is a continuous function of its set of strategies. The operator's objective function is a continuous function of its policy set $\left(P_{b l, x}, P_{t l, x}, P_{r l, x}\right)$. The objective function of a residential user as a follower of the game is a continuous convex function with respect to the set of strategies, and the term of the user's objective function associated with the set of strategies is $f\left(P_{b l, x}, P_{t l, x}, P_{r l, x}\right)$, for which a constant less than 0 is obtained after the second-order partial derivative of the variables. In summary, there is a unique Nash equilibrium solution for the stackelberg game proposed in this paper.

\section{Comprehensive application}

The modified IEEE33 node system is used as a simulation system with one scheduling cycle taken as $24 \mathrm{~h}$ and time interval of $1 \mathrm{~h}$. The data of 3 types of flexible loads are presented in the literature [8]. In order to analyze and compare the role of this master-slave game model in improving the social and economic benefits, three simulation scenarios are set up: the In order to analyze and compare the effect of this master-slave game model in improving social and economic efficiency, three simulation scenarios are set: Scenario 1, price DR does not participate in system dispatch; Scenario 2, price DR participates in system dispatch (no master-slave game model); Scenario 4, price DR participates in system dispatch (with master-slave game model).

Table 1 Cost comparison in different scenarios

\begin{tabular}{|c|c|c|c|c|c|}
\hline Scenes & $\begin{array}{c}\text { Abandoned wind } \\
\text { volume / } \\
(\mathrm{MW} \cdot \mathrm{h})\end{array}$ & $\begin{array}{c}\text { Wind power } \\
\text { consumption rate } \\
/ \%\end{array}$ & $\begin{array}{c}\text { Cost of wind } \\
\text { abandonment penalty } \\
/ \$\end{array}$ & $\begin{array}{c}\text { Power Generation } \\
\text { Costs / \$ }\end{array}$ & $\begin{array}{c}\text { Customer } \\
\text { electricity bill / } \\
\$\end{array}$ \\
\hline 1 & 263.1006 & 129.9018 & 73687.95 & 40904.78 & 119822.4 \\
\hline 2 & 166.3886 & 139.8556 & 42391.57 & 36662.64 & 112677.3 \\
\hline 3 & 146.2926 & 141.928 & 33852.34 & 36155.53 & 110575.1 \\
\hline
\end{tabular}

From the above table, we can see that the change of the load curve in scenario one is not significant compared with the original electric load curve, which is due to the fact that the user-side controllable load accounts for a relatively small proportion and is not highly sensitive to the price of electricity in the calculation case, and the pure electric demand response effect is not ideal. The change of the load curve in Scenario 3 is more obvious and the demand response effect is better, which is due to the use of the multi-energy coupling nature of the user side, the use of price incentives for users to substitute energy use, and the conversion of part of the user's thermal load into electric load. In Scenario 1, the customer has no alternative energy use and the expected revenue from energy sales is high, while in Scenario 2, the customer reduces its cost of electricity purchase through alternative energy use, and the operator's revenue from electricity sales is $4.5 \%$ lower than expected (Scenario 1). In terms of operating costs of the integrated regional energy system, the alternative use of energy by customers resulted in a
$13.5 \%$ increase in wind power consumption rate and a $5 \%$ reduction in generation costs in Scenario 2 compared to Scenario 1. The final overall scenario 2 is $3.8 \%$ less profitable for the DIO than expected (scenario 1). In Scenario 3, the RIO takes into account the impact of the heat and electricity price difference on the customer's energy use, and the energy sales revenue is higher than in Scenario 2 but lower than in Scenario 1, and the final profit in Scenario 3 is the highest. The means to reduce the operating cost of CIES is to increase the proportion of high cost wind power in the total equipment output, and for the strong anti-peak characteristics of wind power in the example, the RIO takes two measures to improve the utilization rate of wind power: one is to install electric heating equipment to convert part of the thermal load into electric load during the peak hours of wind power; the other is to dispatch the load side to make the second is to schedule the load side so that the load curve is as close as possible to the wind power curve, i.e., to "cut the peak and fill the valley" of the load curve. On the one hand, the 
regional integrated energy operator provides incentives for customers to dispatch their curtailable loads and shiftable loads through tariffs, and on the other hand, it provides incentives for customers to dispatch customer-side energy conversion equipment to convert thermal loads into electrical loads and "fill in the valley" for electrical loads through energy price differences, so that the customer's electrical load curve can better follow the wind power output curve and improve the utilization rate of wind power. Wind power utilization rate.

\section{Conclusion}

In this paper, a master-slave game economic model considering new energy consumption and DR uncertainty is proposed. The simulation analysis of the modified IEEE 33-node system concludes that after the introduction of price-based DR, the strategy set corresponding to the Spielberg Nash equilibrium solution obtained by the master-slave game model can effectively reduce the wind power abandonment rate and increase the wind power consumption, so that the operation cost of the distribution network side, the abandonment penalty cost and the loadside customer tariff can be reduced regardless of its response uncertainty. The cost of distribution grid operation, the cost of wind abandonment penalty, and the cost of load-side customer electricity are reduced.

\section{Acknowledgment}

The authors would like to thank the support of the project "the State Grid Corporation Science and Technology Project (SGSDDK00PDJS2000383)".

\section{References}

1. Xu Zheng, Sun Hongbin, Guo Qinglai. A review and outlook of integrated demand response research[J]. Chinese Journal of Electrical Engineering, 2018, 38(24):84-95+336.

2. Aghajani G R, Shayanfar H A , Shayeghi H . Presenting a multi-objective generation scheduling model for pricing demand response rate in micro-grid energy management[J]. Energy Conversion and Management, 2015.

3. Cui X., Zou C. L., Wang H., et al. Optimal scheduling of source-load coordination for combined electric and thermal systems considering wind power consumption[J]. Power Automation Equipment, 2018, v.38;No.291(07):79-86.

4. Li Chunyan, Chen Snap, Zhang Peng,et al. Multitimescale optimal scheduling of demand response accounting for wind power forecast errors[J]. Power Grid Technology, 2018.

5. Hou Jianzhao, Hu Qunfeng, Tan Zhongfu. A multiobjective optimization model for collaborative scheduling of wind power and electric vehicles with demand response $[\mathrm{J}]$. Power Automation Equipment, 2016, 36(007):22-27.
6. Anonymous. Master-slave game-based two-tier bidding strategy for virtual power plants[J]. Power System Automation,2017, 14(v.41;No.612):67-75.

7. Cui Yang, Zhang Huiquan, Zhong Wuzhi, et al. Counting and price-based demand response and wind power consumption day dispatch with CSP power plant participation [J]. Power Grid Technology, 2020, 44(1): 183-191

8. Zeng D, Yao J, Yang S-C, et al. Probabilistic tide calculation accounting for price-based load response uncertainty $[\mathrm{J}]$. Power System Automation, 2015, 39(20): 66-71, 97 .

9. Oprea S, Bâra A . Electricity load profile calculation using self-organizing maps[C]//20th International Conference on System Theory, Control and Computing (ICSTCC) . Sinaia: ICSTCC, 2016: 860-865 .

10. demand response behavior[J] . Power System Technology2017, 41(7): 346-353(in Chinese) . 